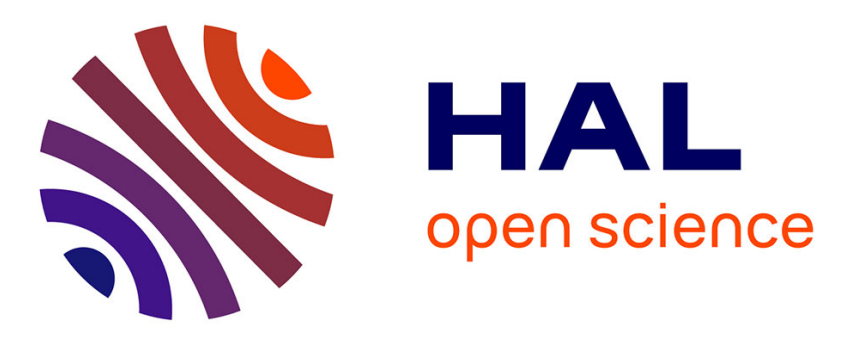

\title{
Neutral modelling of agricultural landscapes by tessellation methods-Application for gene flow simulation.
}

Florence Le Ber, Claire Lavigne, Katarzyna Adamczyk, Frédérique Angevin, Nathalie Colbach, Jean-François Mari, Hervé Monod

\section{To cite this version:}

Florence Le Ber, Claire Lavigne, Katarzyna Adamczyk, Frédérique Angevin, Nathalie Colbach, et al.. Neutral modelling of agricultural landscapes by tessellation methods-Application for gene flow simulation.. Ecological Modelling, 2009, 220 (24), pp.3536-3545. 10.1016/j.ecolmodel.2009.06.019 . hal-00409081

\section{HAL Id: hal-00409081 https://hal.science/hal-00409081}

Submitted on 9 Sep 2009

HAL is a multi-disciplinary open access archive for the deposit and dissemination of scientific research documents, whether they are published or not. The documents may come from teaching and research institutions in France or abroad, or from public or private research centers.
L'archive ouverte pluridisciplinaire HAL, est destinée au dépôt et à la diffusion de documents scientifiques de niveau recherche, publiés ou non, émanant des établissements d'enseignement et de recherche français ou étrangers, des laboratoires publics ou privés. 


\title{
Neutral modelling of agricultural landscapes by tessellation methods - Application for gene flow simulation
}

Texte paru dans Ecological Modelling, doi:10.1016/j.ecolmodel.2009.06.019

\author{
F. Le Ber*, C. Lavigne $\dagger$ K. Adamczyk $\ddagger$ F. Angevin§ \\ N. Colbach, J.-F. Marill and H. Monod**
}

\begin{abstract}
Neutral landscape models are not frequently used in the agronomical domain, whereas they would be very useful for studying given agro-ecological or physical processes. Contrary to ecological neutral landscape models, agricultural models have to represent and manage geometrical patches and thus should rely on tessellation methods. We present a three steps approach that aimed at simulating such landscapes. Firstly, we characterized the geometry of three real field patterns; secondly, we generated simulated field patterns with two tessellation methods attempting to control the value of some of the observed characteristics and, thirdly, we evaluated the simulated field patterns. For this evaluation, we considered that good simulated field patterns should capture characteristics of real landscapes that are important for the targeted agro-ecological process. Real landscapes and landscapes simulated using either a Voronoi or a rectangular tessellation were thus compared when used as input data within a gene flow model. The results showed that neither tessellation method captured field shapes correctly, thus leading to over or (small) under estimation of gene flow. The

\footnotetext{
${ }^{*}$ UMR 7517 LHyGeS \& UMR 7503 LORIA, ENGEES, F-67000 Strasbourg, florence.leber@engees.unistra.fr

${ }^{\dagger}$ UR 1115 PSH, INRA, F-84000 Avignon

‡Unité MIA, INRA, F-78350 Jouy-en-Josas

$\S$ UAR 1240 Eco-innov, INRA, F-78850 Thiverval Grignon

ฯ UMR 1210 BGA, INRA, F-21000 Dijon

"UMR 7503 LORIA, U. Nancy 2, F-54506 Vandœuvre-lès-Nancy

** Unité MIA, INRA, F-78350 Jouy-en-Josas
} 
Voronoi tessellation, though, performed better than the rectangular tessellation. Possible research directions are proposed to improve the simulated patterns, including the use of post-processing, the control of cell orientation or the implementation of other tessellation techniques.

Keyword. Neutral landscape models, agricultural landscapes, gene flow, spatial point process, Voronoi diagrams, rectangular tessellation, field pattern, GenExP-LandSiTes.

\section{Introduction}

Landscape simulation has been a major line of investigation in the fields of ecology and agronomy over the last few years. In landscape ecology, landscape simulation is used for studying the influence of landscape patterns on ecological phenomena [44]. Simulation methods fall into three groups: geostatistical models, neutral models, and process-explicit models [42]. Geostatistical models focus on interpolation methods of spatial data [21]. Processexplicit models include the description of spatial processes and related ecological phenomena which underlie the landscape dynamics (e.g., [48, 33]). In contrast, neutral landscape models (NLM, sensu [17]) provide random landscape structures as a baseline for comparison with real landscape patterns, or for an evaluation of landscape structure effects on ecological processes $[17,16,47]$. Such models are termed neutral as they model no explicit process giving rise to the landscape pattern, they do model totally random or somewhat constrained covering of the area. Neutral models are mainly based on raster approaches, land-use being allocated randomly to pixels and then clustered by various methods (e.g., [42]). Fractal models have also been used [22]. More recently, polygonal approaches, using geometrical tessellations, have been proposed to simulate patchy landscapes $[19,18]$.

Landscape models have been widely used in forest management (e.g., $[33,26])$. In land-use planning and agronomy, such models are less frequent. Spatial models have been used for the simulation of crop allocations on a farm or over a regional territory (e.g., $[28,8]$ ), in order to understand or to plan the organisation of agricultural landscapes according to environmental problems (e.g., water pollution). Closer to ecological approaches, other studies have focused on the links between farming practices and ecological processes with process-explicit models [20,3]. In both cases, the models rely on real spatial data, such as field patterns, landscape characteristics (i.e., soil, slope) or the current agricultural mosaic of a region. As far as we know, 
there are almost no neutral models, such as those developed in landscape ecology, though these models would be very useful when dealing with models of agro-ecological processes. Several reasons for using neutral models can be cited. First, real data are not always available or are too specific and thus reduce the scope of application of the model results. Second, in the case of anthropogenic landscapes, it is necessary to prospect new configurations in order to forecast their effects or to find the best configuration with respect to the given agro-ecological process. Finally, neutral landscapes can be used to test the sensitivity of process models to the spatial variability of agricultural landscapes.

We propose to simulate agricultural landscapes, including both their configuration (the field pattern) and their composition (occurrence of categories of land-use) [32]. However, as mentioned by [20], agricultural landscapes cannot be easily simulated with traditional ecological neutral landscape models for two reasons: (1) they are mainly geometrical, contrarily to less anthropogenic landscapes, and (2) in traditional landscape models, the basic unit is the pixel, and the land-use mosaic (i.e., both the configuration and the composition) only emerges as a result of the simulation (e.g., [48]), whereas in agricultural landscapes, the field pattern is generally stable and "precedes" the land-use. For handling geometric patches as basic units, a straightforward way is to use tessellation methods, such as proposed by [6] or [19]. However, various tessellation methods are available, that result in somewhat different geometries. Good tessellation methods should have the ability to correctly simulate characteristics of real landscapes that are of importance for the targeted agro-ecological process. In the following, we simulate agricultural landscapes using two different tesselation methods. These landscapes are used as input for a gene flow model that handles pollen dispersal as an agro-ecological process. More precisely, we investigate how the values of simulated cross-pollination rates from GM to non-GM fields depend on the tessellation method used to simulate the landscapes, with the rationale that if cross-pollination rates are similar between real and simulated landscapes, this would mean that simulated landscapes capture essential features of real landscapes. To measure and compare these features is necessary to interpret safely the results of gene flow models on simulated landscapes. Whereas various tessellation methods have been used as a basis for landscape generation (e.g., $[6,18]$ ), as far as we know, very few studies focused on their evaluation via their impact on the outcome of ecological processes (e.g., [23]).

To this end, we followed a two-step procedure: we first generated simulated landscapes with basic properties of actual landscapes; then, we as- 
sessed the impact of the tessellation methods on gene flow. In Section 2, we describe tessellation methods and an innovative point-process model which can be used for generating tessellation seeds. Section 3 focuses on the combination of tessellation methods and the gene flow model. Results are given in Section 4 and they are discussed in Section 5. The conclusion proposes some promising leads for improving the realism of tessellation-based simulated landscapes.

\section{Neutral models of agricultural landscapes}

In this section, we focus on the simulation of agricultural landscapes in terms of configuration (field shape, size, and location) and composition (the land-use mosaic). The configuration has been designed with the help of tessellation methods. The composition can be obtained in a purely random manner, or on the basis of expert knowledge or real data. Furthermore, our objective was to explore the variability of landscape patterns on the basis of some given, controlable parameters describing the basic properties of actual agricultural landscapes. We therefore sought a correspondence between geometrical tessellations and spatial patterns of existing landscapes.

\subsection{Simulating the geometry of agricultural landscapes}

Tessellation entails filling a defined area with geometrical figures (hereafter refered to as cells) without any overlapping and leaving no holes. Tessellation methods are based on spatial statistics and computational geometry [38]. They are used in several fields of study, as in image recognition or geography for example, but more for spatial analysis than for simulation (e.g., [15]). Purely random or regular tessellations were not taken into consideration, and we have focused on approaches that allow the handling of tessellation in terms of given characteristics of real landscapes. These approaches are based on a tessellation algorithm and a given set of points or segments, called the tessellation seeds.

The ability of a tessellation method to preserve the features observed in a real landscape can be evaluated, on the one hand, on the basis of the geometry of the tessellation cells (size, shape, orientation), and, on the other hand, on the basis of their spatial distribution (clustered or regular patterns). In both tessellation methods investigated here, the geometry of the cells is completely determined by the tessellation algorithm. In contrast, the spatial distribution of tessellation cells depends on the distribution of the tessellation seeds. Consequently, a tessellation which is based on the 
"seeds" of a real landscape will preserve the spatial configuration of the fields in that landscape.

\subsubsection{Simulation of tessellation seeds}

If tessellation seeds are to be generated in a stochastic manner, they can be considered as a realisation of a spatial (2D) point-process, within a bounded window. Furthermore, if the seeds correspond to specific points of a landscape pattern, then the point-process should fit the spatial distribution of these specific points. Eventually, if examples of such specific points are available, it is possible to estimate the point-process parameters in order to simulate similar configurations of seeds.

We describe here an example of a point-process fitted to centroid patterns (see [1] for more details). We chose a point-process that could yield an aggregated or regular centroid pattern and account for the fact that the distance between a pair of real centroids is greater than a certain threshold, proportional to the minimal field area. The Poisson process, commonly used as the reference point-process, is not appropriate for modelling centroid distribution. The pairwise-interaction process (see for example [45]) is more suitable for our purposes. The model controls both the mean number of points per unit area (intensity) and the distribution of pairwise distances between the points. It is defined by the following density function:

$$
f(x ; \lambda, \theta) \propto \lambda^{n} \prod_{i<j} \gamma_{\theta}\left(\left\|x_{i}-x_{j}\right\|\right)
$$

where $x_{1}, \ldots, x_{n}$ are the coordinates of $n$ centroids, $\lambda$ is the intensity of the process and $\gamma_{\theta}(\cdot)$ is the interaction function depending on the Euclidean distance between the pairs of points and the interaction parameter $\theta$. For $\gamma_{\theta} \equiv 1$ the model is reduced to a homogeneous Poisson process with intensity $\lambda$ : no interaction between the points is observed. The values of $\gamma_{\theta}>1$ introduce an aggregated point pattern and the values of $\gamma_{\theta}<1$ introduce a regular point pattern at a given distance.

\subsubsection{Two tessellation methods}

The Voronoi tessellation Given a set of points in the Euclidean plan, the set of seeds, a Voronoi tessellation -or Voronoi diagram- is a covering of the Euclidean plan with non-overlapping convex polygons, each surrounding a seed. The points in a polygon are closer to its seed than to any other seed located in any other polygon. The edges therefore consist in the points 
located at an equal distance from two seeds (Figure 1(a)). The Delaunay triangulation [38] can be used to determine the polygons.

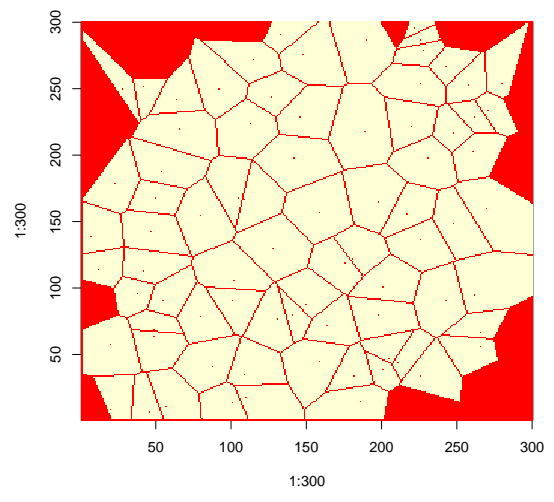

(a) Voronoi tessellation

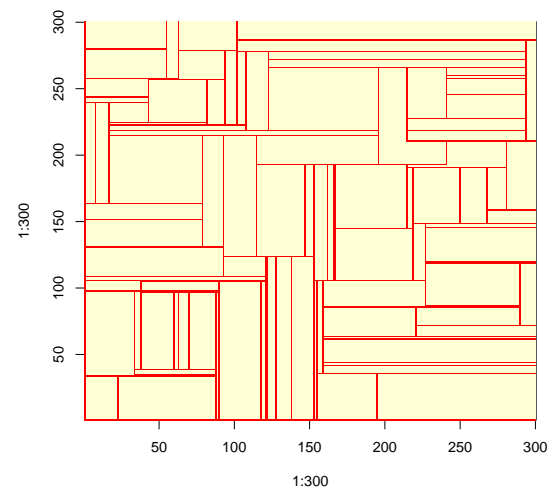

(b) rectangular tessellation

Figure 1: Two different tessellations based on sets of seeds (region A1, scale 1/5): the Voronoi tessellation (a) and the rectangular tessellation with only T-vertices (b). Infinite Voronoi cells were eliminated.

The major advantage of this tessellation is the correspondence between the pair polygon/seed and the pair field/centroid. For example, equally distanced seeds yield regular pattern of cells, aggregated seeds yield clusters of small cells and aligned seeds result in range-structured cells. Similarly, the configuration of field centroids characterises the spatial distribution of the fields: for instance, an aggregated pattern of centroids reveals clusters of small fields. Therefore, a simulation based on statistic parameters that are estimated on centroids of real landscapes is possible. The drawbacks of this tessellation are related to the shape of the polygons which are convex and often have a higher number of vertices than actual fields.

A rectangular tessellation In a rectangular tessellation, the area is filled in with non-overlapping rectangles. We eliminated the simple case when the rectangles are defined by two orthogonal sets of parallel lines and considered only those rectangles sharing T-vertices (Figure 1(b)). In such tessellations, a vertex of a rectangle cannot belong to more than three rectangles (no $\mathrm{X}$-vertex). A method for building such tessellations has been described in [34]. The basic principles of the algorithm is to generate the edges of the 
rectangles by crossing two-directional rays starting from a set of points.

In this case, there is no obvious correspondence between the rectangles and the fields, since the basic geometric unit is a segment rather than a polygon and a seed is located anywhere along an edge. We found no specific points representative of a real landscape to be used as seeds for the rectangular tessellation. Nevertheless, the geometry of the rectangular cells yields a priori more realistic field shapes (for at least some regions, see figure $3(\mathrm{~b})$ ) than the Voronoi cells, and certain overall characteristics of a landscape pattern can be conserved: for example, the number of the polygons is equal to the number of seeds plus one.

\subsection{Simulating the land-use mosaic of agricultural landscapes}

The simulation of the land-use mosaic can be handled in several ways. Basically, in most landscape models, crops are randomly allocated according to a probability distribution: e.g., in binary patchy landscapes, each plot is grown with a crop according to a probability $p$ (vs. $1-p$ ) and its own surface area. When several crops are under consideration, each crop is associated with a probability value $p_{c}$, so that $\sum_{c} p_{c}=1$.

Methods have been proposed to improve the purely random approach. In [19], a Gibbs process is introduced to model the interactions between pairs of neighbouring patches. According to the parameter values of this model, the landscape mosaic can be more or less heterogeneous. Other processes could be used, based on the statistical study of spatial and temporal patterns of real field mosaics $[9,10]$. Furthermore, stochastic neighbourhood rules can be learnt from a real dataset, as proposed in [35], where Hidden Markov Models are applied on land-use temporal and spatial data.

\subsection{The GenExP-LandSiTes software}

GenExP-LAndSiTes ${ }^{1}$ is a software simulating two-dimensional agricultural landscapes. It is written in Java, and it is freely accessible through a Gnu Public Licence [30]. Its main features fall into three categories: the generation of the field patterns based on the simulation of spatial pointprocesses and on tessellation methods; the post-processing that improves the realistic aspect of the simulated landscape; and the computation of landscape statistics.

The two tessellation methods described above have been implemented: the Voronoi tessellation, using the "3D Hull" algorithm for Delaunay tri-

\footnotetext{
${ }^{1}$ LANDscape SImulation based on TESsellation.
} 
angulation [39] and the rectangular tessellation, using the Mackisack algorithm [34]. The land-use mosaic is built randomly according to a chosen distribution of land-uses. GenExP-LandSiTes was also coupled with the statistics $\mathrm{R}$ software to have high-level tools for the spatial point-process simulation [5]. Finally, GenExP-LandSiTes provides a library to calculate basic landscape descriptors (field area, perimeter, number of vertices, centroid and shape).

\section{Application to the study of gene flow}

In the case of coexisting GM, conventional and organic crops, two agronomic aspects have to be taken into account. The first aspect concerns the cropping of several agricultural species bearing the same transgene (for instance a tolerance to the same herbicide). Interactions between these different crops in a cropping system must be studied to define the best management of volunteers and avoid selection or breakdown of this particular gene.

The second aspect concerns the cultivation of GM and non-GM varieties of the same species for which, in Europe, coexistence rules between GM and non-GM crops (e.g., minimum distances between crops) have to be implemented to respect harvest impurity thresholds of non-GM crops, e.g., less than $0.9 \%$ of GM seeds in non-GM harvests (2003/556/EC, 1830/2003/EC). Several coexistence studies have been carried out to address this issue (see for instance: [7, 43, 41, 36]). Few of them have dealt with the impact of the distribution of GM and non-GM crops in the landscape [36, 31]. Moreover, they were based on real agricultural landscape which limits the number of studied cases (due to the accessibility of spatialised data). Only a few recent studies were based on simulated landscapes $[11,27,13]$.

\subsection{Combining landscape models with gene flow models}

The GenExP-LandSiTes software was combined with MaPod-maize-a spatially explicit pollen dispersal model [2]- and we assessed the influence of landscape structures on maize gene flow. The investigation entailed the following four-step process (figure 2).

\subsubsection{Generation of field patterns}

Three French landscapes were chosen for this study (Figure 3). The landscapes, restricted to a $1.5 \mathrm{~km} \times 1.5 \mathrm{~km}$ window, differed mainly in mean 


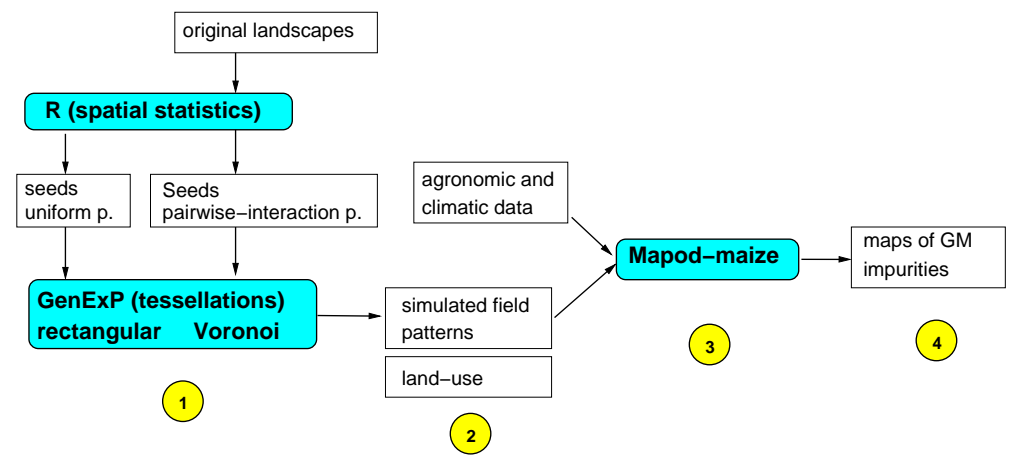

Figure 2: Combination process of the GenExP-LAndSiTes and Mapodmaize softwares: (1) generation of field patterns, (2) crop allocation, (3) pollen dispersal, and (4) GM impurity rates.

plot area : small irregular plots (1 ha) in region $P 1$ (French region MidiPyrénées), medium and (rather) elongated plots (2 ha) in region $A 1$ (French region Alsace), and large and (rather) compact plots (3.5 ha) in region $S_{4}$ (French region Centre).

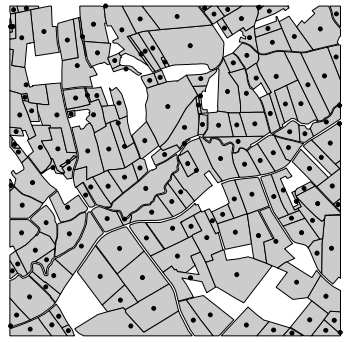

(a) P1

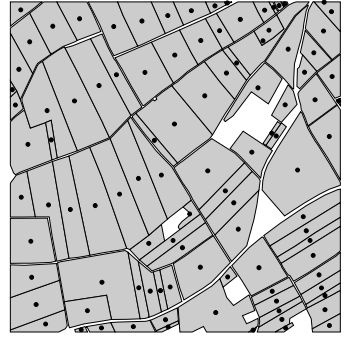

(b) A1

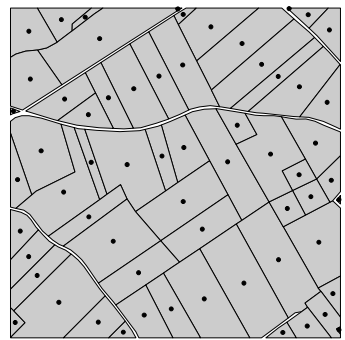

(c) $\mathrm{S} 4$

Figure 3: Three agricultural landscapes with plot centroids.

For each region, the pairwise-interaction model (section 2.1.1) was fitted to the pattern of field centroids (see [1] for details). The parameters of the pairwise-interaction model were estimated using the maximum pseudolikelihood method proposed by [4]. The seeds of Voronoi tessellations were generated according to the fitted pairwise-interaction process ${ }^{2}$ whereas the seeds of rectangular tessellations were generated as independent unifor-

\footnotetext{
${ }^{2}$ We used the Metropolis-Hasting algorithm, implemented in the R-spatstat library [5].
} 
mally distributed random points. ${ }^{3}$ From each original landscape, ten virtual field patterns were designed using the GENExP-LANDSITES software, five based on the Voronoi tessellation and five based on the rectangular tessellation. Overall, thirty virtual field patterns were generated. ${ }^{4}$ Taking into account the original patterns, there were 33 field patterns. Landscapes were simulated within a $1.5 \mathrm{~km} \times 1.5 \mathrm{~km}$ window without any predefined hole (no non-agricultural zone, see Figure 1).

\subsubsection{Crop allocation to fields}

The total proportion of field area allocated to maize was set at $70 \%$, thus simulating French production areas where maize is a major crop. The proportion of GM maize within this area was set to either $10 \%$ or $50 \%$, simulating low and high acceptance of GM maize, respectively. GM and non-GM maize were randomly attributed to the fields in each of the $1.5 \times 1.5$ maps until the desired proportions were met. Three replicated allocations of crops were performed per map. Overall, we thus produced 198 crop allocations (33 field patterns x 2 proportions of GM maize x 3 replicates).

\subsubsection{Pollen dispersal}

MAPOD-maize simulates pollen dispersal through a quasi-mechanistic approach that takes into account crop phenology and various agronomic and climatic conditions [2]. The sub-model for pollen dispersal was adapted from [25] and it accounts for distance between source and receptor plants as well as wind direction and intensity, and as plant height. To focus on the impact of spatial characteristics of landscapes, we kept agronomic and climatic inputs constant and identical for the GM and the conventional varieties. In particular, both varieties were assumed homozygous with the same pollen production and synchronous flowering. Wind was supposed moderate $(3 \mathrm{~m} / \mathrm{s})$ and orientated from west to east to simulate common French situations.

\subsubsection{GM impurity rates}

GM impurity rates were calculated in each non-GM field as the proportion of seeds containing at least one copy of the transgene at harvest. For each simulated crop allocation, we also calculated the proportion of fields with impurity rates exceeding either $0.1 \%$ or $1 \%$.

\footnotetext{
${ }^{3}$ Number of seeds $=$ number of fields in the original pattern minus 1.

${ }^{4} 3$ original patterns $\times 5$ simulations $\times 2$ tessellation methods.
} 


\subsection{Statistical analyses}

\subsubsection{Assessing the impact of the tessellation method on land- scapes characteristics}

[40] showed that six indices are sufficient to characterize landscape patterns. Since we were not interested in fractal landscapes, land-use or scaling, only two remained: the average patch compaction (i.e., perimeter/area), and the average patch shape (perimeter $/ \sqrt{\text { area }}$ ). In the case of simple convex shapes, as produced by the Voronoi and rectangular tessellations, the average patch compaction is highly correlated to the average patch area. The areas (in hectares) and the shapes $\left(I_{S}=\right.$ perimeter $\left./ 4 \sqrt{\text { area }}\right)$ of fields were thus calculated over each of the 33 field patterns. To assess the within-landscape variability of field areas and shapes, we also calculated the standard deviation of these measurements within field patterns. Although statistical tests on simulated data should be carefully considered, means and standard deviations of area and shape index at the field pattern level were submitted to analyses of variance using original landscape (qualitative, three levels), tessellation method (three levels: none=original landscapes, Voronoi, and rectangular) and their interaction as factors. Field areas and standard deviations of the shape index within field patterns were log-transformed to improve normality of residuals.

Besides the geometry of the simulated fields, we were also interested in their spatial distribution. We evaluated this distribution by examining the distances between field centroids, using the nearest neighbour distance distribution function $G$. For a given distance $r$ the value $G(r)$ gives the probability that the nearest-neighbour of an arbitrary centroid lies within this distance.

\subsubsection{Assessing the impact of tessellation method on GM impu- rity rates}

Impacts on GM impurity rates for landscape type and tessellation method were assessed through analyses of variance and comparisons of least-squares means. Fixed effect used in the model were original landscape, tessellation method, proportion of GM crop (qualitative, two levels) and their two-way interactions. To account for correlations among the five simulations performed with each tessellation for each original landscape, a repet factor was declared as random and nested in (region, tessellation, GM proportion). Similarly, to account for correlations among fields within a single crop pattern, allocation nested in (repet, region, tessellation, GM proportion) was 
also declared as random. All GM impurity rates were log-transformed prior to analyses to improve the normality of residuals. The same model was applied to the proportions of fields exceeding the $0.1 \%$ and $1 \%$ threshold except that the allocation term was not needed because proportions are response variables defined at the landscape level. Prior to analyses, square roots of proportions were arcsine-transformed to homogenise variances.

\section{Results}

\subsection{Comparison of original and simulated field patterns}

The number of fields (Table 1) was fixed for the rectangular tessellation, since we used a fixed number of seeds. On the contrary, for the Voronoi tessellation, the number of seeds is a random variable whose mean value should be close to the number of observed centroids: in our case it appeared to be slightly lower. One explanation is that the Voronoi tessellation produced infinite cells on the landscape borders that were eliminated (cf. Figure 1).

Field areas and shapes were not affected by the same factors: whereas field areas and their within-landscape variability were mostly explained by the original landscape, field shapes and their variability were mostly explained by the tessellation method. Variability due to small random variations of seed distribution among replicate patterns was small (variance estimates in Table 2). Average field areas (Table 1) with the rectangular tessellations were close to the ratio landscape area/number of original fields. Actually, the rectangular tessellation is a total covering of the initial window. For the same number of fields, the original landscapes had a lower average field area since they contained non-agricultural areas. The results of the Voronoi tessellations were slightly more variable. In contrast, the variability of field areas was lower for the Voronoi tessellations than for both real landscapes and rectangular tessellations. Rectangular tessellations had the highest variability. Regarding field shapes (Table 1), as expected, each tessellation gave homogeneous results with respect to the three original landscapes, but somewhat different from the the original field shapes. Indeed, the shape index of rectangular fields was too high (elongated shapes) while the Voronoi fields were too compact. Furthermore, the within-landscape variability of shapes was very high for the rectangular tessellation and somewhat low for the Voronoi tessellation.

Regarding the spatial distribution of fields, results for region A1 are given in Figure 4. The estimates of the $G$ function for the Voronoi simulations were close to the original pattern. Indeed, the seed generation in Voronoi 


\begin{tabular}{|c|c|c|c|}
\hline & $\mathrm{P} 1$ & A1 & $\mathrm{S} 4$ \\
\hline & \multicolumn{3}{|c|}{ Average number of fields } \\
\hline Voronoi & $150.4 \pm 8.7$ & $96.4 \pm 12.8$ & $45.8 \pm 4.9$ \\
\hline Rectang. & 175 & 100 & 63 \\
\hline \multirow[t]{2}{*}{ Original } & 175 & 100 & 63 \\
\hline & \multicolumn{3}{|c|}{ Average field area (ha) } \\
\hline Voronoi & $1.28 \pm 0.08$ & $2.00 \pm 0.23$ & $4.08 \pm 0.40$ \\
\hline Rectang. & $1.3 \pm 0.04$ & $2.24 \pm 0.07$ & $3.68 \pm 0.12$ \\
\hline \multirow[t]{2}{*}{ Original } & 1.09 & 2.05 & 3.55 \\
\hline & \multicolumn{3}{|c|}{ Standard deviation of field area (ha) } \\
\hline Voronoi & $0.51 \pm 0.02$ & $1.08 \pm 0.2$ & $1.65 \pm 0.36$ \\
\hline Rectang. & $1.15 \pm 0.09$ & $1.99 \pm 0.11$ & $3.17 \pm 0.32$ \\
\hline \multirow[t]{2}{*}{ Original } & 0.91 & 1.84 & 2.79 \\
\hline & \multicolumn{3}{|c|}{ Average shape index } \\
\hline Voronoi & $1.32 \pm 1.810^{-3}$ & $1.34 \pm 0.610^{-3}$ & $1.32 \pm 3.310^{-3}$ \\
\hline Rectang. & $1.70 \pm 0.12$ & $1.75 \pm 0.11$ & $1.73 \pm 0.19$ \\
\hline \multirow[t]{2}{*}{ Original } & 1.50 & 1.53 & 1.48 \\
\hline & \multicolumn{3}{|c|}{ Standard deviation of shape index } \\
\hline Voronoi & $0.10 \pm 1.210^{-2}$ & $0.13 \pm 1.410^{-2}$ & $0.11 \pm 2.710^{-2}$ \\
\hline Rectang. & $0.91 \pm 0.12$ & $0.97 \pm 0.15$ & $0.98 \pm 0.18$ \\
\hline Original & 0.31 & 0.28 & 0.19 \\
\hline
\end{tabular}

Table 1: For each original landscape (P1, A1, S4) and each tessellation method: average number of fields, field areas, and field shapes (mean \pm standard error); variabilities of field areas and field shapes measured as within-landscape standard deviation (mean \pm standard error).

simulations was based on a pairwise-interaction process fitted to the real centroids, and thanks to the tessellation method, the centroids of tessellation cells are close to the seeds. On the contrary, the estimates of the $G$ function for rectangular tessellation differed from the original pattern. Indeed, the rectangular tessellation did not allow us to link the centroids of the simulated rectangles to the centroids of the actual landscapes.

\subsection{Impact of the tessellation method on GM impurity rates}

As expected, the proportions of GM maize over the landscape had the highest effect on impurity rates (Tables 3 and 4). The impact of the tessellation method was comparable to that of the original landscape (close F-values) 


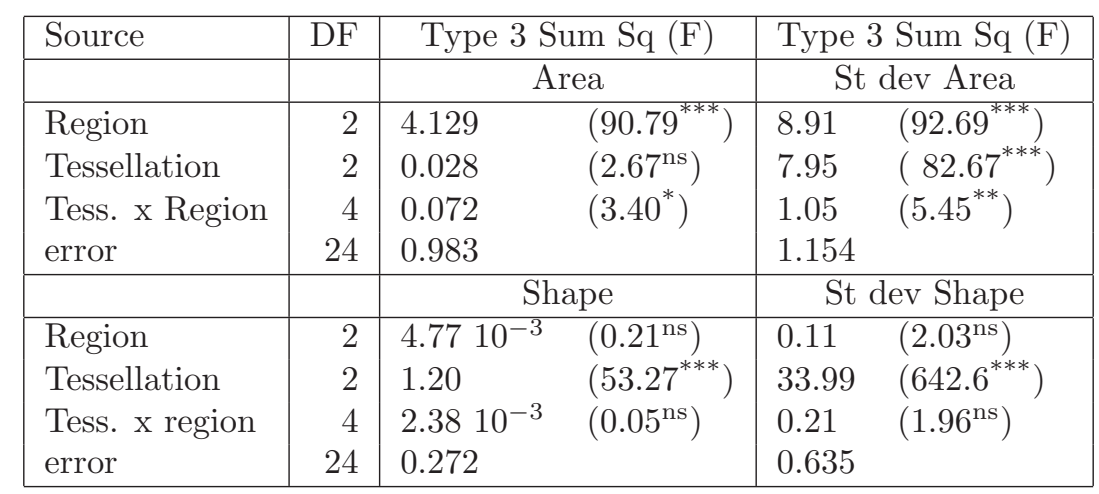

Table 2: Analysis of variance on characteristics of original and simulated field patterns (DF: degrees of freedom; Sum Sq: sum of squares; F: Fisher statistics; St dev: standard deviation; ${ }^{\text {ns }}: P>0.05 ;{ }^{*}: P>0.01{ }^{* *}$ : $\left.P>0.001 ;{ }^{* * *}: P<0.001\right)$. The $\mathrm{F}$ tests were performed assuming the mixed model described in the main text.

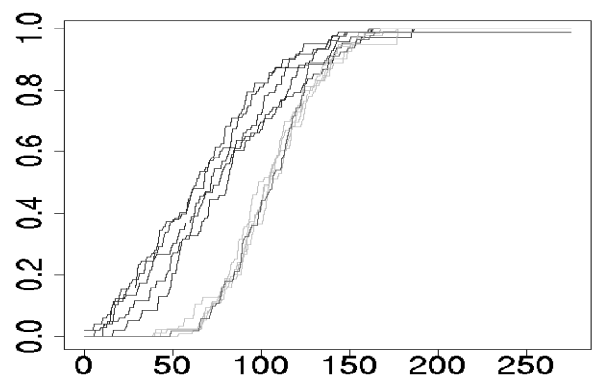

Figure 4: Estimate of $G$ function for the field centroids of pattern A1. Red line: original pattern. Gray lines: five Voronoi simulations. Blue lines: five rectangular simulations. X-axis: distance $(\mathrm{m})$ between two nearest neighbours.

when either individual field cross-pollination rate or proportion of fields above the $0.1 \%$ threshold were considered. Differences between the tessellation methods decreased largely when the $1 \%$ threshold value was considered and became much smaller than differences between original landscapes (Table 4). The impact of the tessellation method did not differ much between regions (small $\mathrm{F}$-value for the Tessellation $\mathrm{x}$ Region interactions).

The large effect of the tessellation method was mainly due to differences between Voronoi and rectangular simulated landscapes. Values for real landscapes were generally close to those on simulated Voronoi landscapes: pairwise comparisons of least-squares means indicate that Voronoi tessellations resulted in impurity rates closer to those simulated on original 


\begin{tabular}{|l|r|r|}
\hline Effect & DF & \multicolumn{1}{|c|}{ F } \\
\hline Tessellation & 2 & 7.20 \\
Region & 2 & 8.53 \\
GM proportion & 1 & 319.46 \\
Tessellation x region & 4 & 1.54 \\
Tessellation x GM prop. & 2 & 2.30 \\
Region x GM prop. & 2 & 0.47 \\
\hline \hline Error variances & Estimation & St dev \\
\hline repet in (tess. x region x GM prop.) & 0.01 & 0.04 \\
alloc in (repet. x tess. x region x GM prop.) & 0.38 & 0.06 \\
Residual & 4.20 & 0.06 \\
\hline
\end{tabular}

Table 3: Analysis of variance with fixed and random effects (Proc Mixed in SAS 8.01, SAS Institute, Cary, NC, USA) on individual field $\left(\log _{10}\right)$ GM impurity rates (DF: degrees of freedom; F: Fisher statistics; St dev: standard deviation). All Fisher statistics are calculated using the repet in (tess. $\mathrm{x}$ region x GM prop.) variance as error variance (52 degrees of freedom).

landscapes than rectangular tessellations. ${ }^{5}$ The same result was observed in terms of the proportions of fields exceeding the $0.1 \%$ threshold. ${ }^{6}$ As expected from the small $\mathrm{F}$-value, outputs of simulations were very close for the two tessellation methods and the original landscape when the $1 \%$ threshold was considered. ${ }^{7}$

\section{Discussion}

Methods have been investigated for modelling 2D-geometrical landscapes, such as a distribution of fields over an area, maintaining certain characteristics of the original landscapes. The rationale for doing so lies in the recent increasing interest in spatially explicit modelling that, to some extent, is due to the increased availability of land-use maps through aerial or satellite pictures. Relating landscape characteristics (e.g., hedgerow density) to ecological (e.g., spread of an invasive species) or physical (e.g., water pollution) processes has been increasingly frequent. However, understanding how landscape structures influence the observed spatial patterns requires more

\footnotetext{
${ }^{5} \mathrm{LS}$ mean $\pm \mathrm{sd} \log _{10}$ (impurity rate): original $=-3.55 \pm 0.14$; Voronoi $=-3.51 \pm 0.06 ;$ rectangular $=-3.18 \pm 0.06$

${ }^{6} \mathrm{LS}$ mean $\pm \mathrm{sd}$ of arc sinus(proportion above $\left.0.1 \%\right)^{1 / 2}$ : original $=0.88 \pm 0.04$; Voronoi $=0.90 \pm 0.017 ;$ rectangular $=0.98 \pm 0.017$

${ }^{7} \mathrm{LSmean} \pm \mathrm{sd}$ arc sinus (proportion above $\left.1 \%\right)^{1 / 2}$ : original $=0.52 \pm 0.02 ;$ Voronoi $=0.49 \pm 0.01 ;$ rectangular $=0.51 \pm 0.01$
} 


\begin{tabular}{|c|c|c|c|c|}
\hline Threshold & & $1 \%$ & $0.1 \%$ & \\
\hline Effect & $\mathrm{DF}$ & $\mathrm{F}$ & $\mathrm{F}$ & \\
\hline Tessellation & 2 & 1.52 & 6.64 & \\
\hline Region & 2 & 9.01 & 6.95 & \\
\hline GM proportion & 1 & 567.02 & 445.43 & \\
\hline Tessellation $\mathrm{x}$ region & 4 & 1.40 & 0.58 & \\
\hline Tessellation x GM proportion & 2 & 0.73 & 0.00 & \\
\hline Region x GM proportion & 2 & 4.83 & 1.57 & \\
\hline Threshold & \multicolumn{2}{|c|}{$1 \%$} & \multicolumn{2}{|c|}{$0.1 \%$} \\
\hline Error variances & Estimation & St dev & Estimation & St dev \\
\hline repet in (tess. $\mathrm{x}$ region $\mathrm{x}$ GM prop.) & $2.3610^{-4}$ & $7.8010^{-4}$ & $3.3910^{-3}$ & $1.7610^{-3}$ \\
\hline Residual & $95.9910^{-4}$ & $11.8210^{-4}$ & $15.0910^{-3}$ & $1.8610^{-3}$ \\
\hline
\end{tabular}

Table 4: Analysis of variance with fixed and random effects (Proc Mixed in SAS 8.01, SAS Institute, Cary, NC, USA) on proportions of fields above a given threshold of impurity rate (DF: degrees of freedom; F: Fisher statistics; St dev: standard deviation). All Fisher statistics are calculated using the repet in (tess. $\mathrm{x}$ region $\mathrm{x}$ GM prop.) variance as error variance (52 degrees of freedom).

than data from a few maps, because each map is unique and differs from others in many ways. Coupling landscape models to process-explicit models (such as the here-used model for pollen dispersal) provides a means to explore the impact on a given process of small random variations in landscape characteristics (replication) as well as large systematic differences of these characteristics.

Whereas neutral landscape models are frequent in ecology [44, 47], tessellation methods have been rarely used. Furthermore, we know of no other landscape model exploring variation in the geometry of fields except that of [18]. Such an approach means that the impact of the geometry of agricultural landscapes on agro-ecological processes can be investigated. It also allows crop allocations over similar (but not identical) geometrical field patterns to be varied. The difficulty is in controlling important landscape characteristics, here for example field areas and shapes or distances among field centroids, while allowing other characteristics to vary.

\subsection{Comparing tessellation methods w.r.t. original land- scapes}

Neither simulation method really succeeded in providing replicates of real landscapes that would allow studying the impact of small random variations 
in landscape geometry. While both tessellation methods allowed simulation of landscapes with numbers of fields and average field areas that resembled those of the original landscapes, neither correctly reflected the shape and most of all the within-landscape variability of this shape: the Voronoi tessellation produced somewhat too compact fields with low variability while the rectangular tessellation produced overly elongated fields with an overly high variability. Systematic post-processing, e.g., deleting too small/elongated cells (in the case of the rectangular tessellation) or deleting vertices in order to deform shapes (in the case of the Voronoi tessellation) could be a solution to improve suitability of tessellation results with the original landscapes.

As mentioned in Section 2.1, the a priori major advantage of the Voronoi tessellation over the rectangular tessellation for simulating agricultural landscapes is that a correspondence can be established between the pair polygon/seed and the pair field/centroid. This property is not exact, especially for concave fields, but it proved efficient to simulate patterns where field centroids respect certain constraints, e.g., a minimum distance between two centroids. Indeed, using the pairwise-interaction point-process (see Section 2.1.1) we produced Voronoi tessellations with similar distributions of nearest-neighbour distances between centroids as the original landscapes. Other characteristics of landscapes have to be investigated. For example, in the rectangular tessellation, we could take advantage of the growing directions of the segments in order to simulate the average orientations of fields.

However, both the Voronoi and the rectangular tessellations start with the distribution of a set of points. This step is completely artificial in the context of real landscape formation and makes a model fit rather difficult. One possible improvement consists on studying the tessellation models based on the geometrical units naturally present in an agricultural landscape: polygons or lines. In a recent work, [18] proposed to use the Delaunay triangles (rather than the Voronoi polygons) and to aggregate them according to linear networks. Furthermore, line-based tessellations, such as proposed by [37], can also be explored to simulate the geometry of agricultural landscapes.

\subsection{Comparing pollen flows w.r.t the characteristics of land- scapes}

A recent spatial sensitivity of GM impurity rates that also used the MAPOD-maize software has concluded that field geometry was a minor factor affecting impurity rates in comparison to maize frequency and spatial ar- 
rangement [46]. Our results confirm this: GM maize proportion was the main factor explaining impurity rates. However, our simulations highlighted some influence of field geometry: GM impurity rates obtained using the Voronoi tessellations tended to be a little under-estimated, while those from the rectangular tessellation tended to be over-estimated, in particular more fields exhibited impurity rates that exceeded $0.1 \%$. This difference might be explained both by the larger variability in field area as well as by the elongated field shapes in the rectangular tessellation. Indeed, in simulations that only took into account one source and one recipient field, large compact recipient fields such as those simulated by the Voronoi tessellation have been shown to be the least susceptible to incoming gene flow [14, 24]. This property is likely due to a larger average distance of points to field edges, this distance being maximum within Voronoi polygons.

In general, impurity rates obtained with Voronoi tessellations were close to those simulated on real landscapes. This is to some extent due to the choice of real landscapes, two of them being covered by regular and mostly large fields, but it is also likely to be a general result as small elongated fields are less and less frequent in current landscapes due to mechanisation and land consolidation. On the contrary, landscapes with regular compact fields are more and more the case. As a first step, Voronoi tessellation thus appears a good basis for simulating landscapes for empirical gene flow models such as MAPOD-maize.

\subsection{Simulating land-use}

In this paper, we only simulated three land-uses (conventional maize, GM maize, others) that were allocated randomly within the landscapes. Characteristics of the fields, such as the area or the neighbourood, were not taken into account. Furthermore, we only needed a 1-year crop simulation, since maize pollen or seeds do not persist under European climatic conditions.

To investigate the effect of landscape structure and land-use on other crops such as rapeseed [12], temporal (succession of land-uses) and spatial (surface or neighbouring crops) constraints should be taken into account. This is possible thanks to the underway coupling of GenExP-LAndSiTeS with the data-mining software CARROTAGE [29].

\section{Conclusion}

When studying agro-ecological or physical processes such as gene flow, erosion, and spore dispersal, in an agricultural landscape, a large number of 
fields and their spatial organisation must be considered, and therefore, experimentation under field conditions is complicated and expensive, if not impossible. Computer simulations are less costly and dramatically increase the number of configurations that can be investigated. Furthermore, there is still little landscape data available, and the extrapolation of the results obtained in a few specific contexts is not easy. Thus, it is necessary to simulate neutral agricultural landscapes in order to explore the variability of landscape characteristics from which more general conclusions can be drawn. In this paper, we tested two tessellation methods with respect to gene flow. Granted, neither of the methods fully captured field shapes of real landscapes and therefore they only partly reached the given objectives. Indeed, the two methods either over-estimated (for the rectangular tessellation) or under-estimated (slightly) gene flow (for the Voronoi tessellation). However, the results have provided promising leads for further developments. We shall have to experiment other process models and improve tessellation methods for a better control of their behaviour with respect to the characteristics of actual landscapes. Possible research directions should include the use of post-processes (i.e., deletion of small cells, fusion of vertices), the control of cell orientations (rectangular tessellation), or the implementation of other tessellation techniques (e.g., line-based tessellations).

\section{Acknowledgments}

Original maps were provided by the Institute for the Protection and Security of the Citizen (Joint Research Centre of the European Union) and AUP (Agence Unique de Paiement / French Payment Agency CAP Support). We acknowledge support from the French national program "ACI OGM et environnement". We wish to thank Ms S. Tanis-Plant for fruitful discussion and advice in English.

\section{References}

[1] Katarzyna Adamczyk, Frédérique Angevin, Nathalie Colbach, Claire Lavigne, Florence Le Ber, and Jean-François Mari. GenExP, un logiciel simulateur de paysages agricoles pour l'étude de la diffusion de transgènes. Revue Internationale de Géomatique, 17(3-4):469-487, 2007.

[2] F. Angevin, E. K. Klein, C. Choimet, A. Gauffreteau, C. Lavigne, A. Messéan, and J.-M. Meynard. Modelling impacts of cropping sys- 
tems and climate on maize cross pollination in agricultural landscapes: The MAPOD model. European Journal of Agronomy, 28(3):471-484, apr 2008.

[3] S. Aviron, P. Kindlmann, and F. Burel. Conservation of butterfly populations in dynamic landscapes: The role of farming practices and landscape mosaic. Ecological Modelling, 205:135-145, 2007.

[4] A. Baddeley and R. Turner. Practical maximum pseudolikelihood for spatial point patterns. Australian and New Zealand Journal of Statistics, 42(3):283-322, 2000.

[5] A. Baddeley and R. Turner. Spatstat: an R package for analyzing spatial point patterns. Journal of Statistical Software, 12(6):1-42, 2005.

[6] T.M. Barrett. Voronoï tessellation methods to delineate harvests units for spatial forest planning. Canadian Journal of Forest Research (Revue Canadienne de Recherche Forestière), 27:903-910, 1997.

[7] A.-K. Bock, K. Lheureux, M. Libeau-Dulos, H. Nilsagård, and E. Rodriguez-Cerezo. Scenarios for co-existence of genetically modified, conventional and organic crops in european agriculture. Technical report, European Comission, 2002.

[8] G. J. Carsjens and W. van der Knaap. Strategic land-use allocation: dealing with spatial relationships and fragmentation of agriculture. Landscape and urban planning, 58:171-179, 2002.

[9] M.S. Castellazzi, J.N. Perry, N. Colbach, H. Monod, K. Adamczyk, V. Viaud, and K.F. Conrad. New measures and tests of temporal and spatial pattern of crops in agricultural landscapes. Agriculture, Ecosystems ES Environment, 118:339-349, 2007.

[10] M.S. Castellazzi, G.A. Wood, P.J. Burgess, J. Morris, K.F. Conrad, and J.N. Perry. A systematic representation of crop rotations. Agricultural Systems, 97:26-33, 2008.

[11] G. Ceddia, M. Bartlett, and C. Perrings. Landscape gene flow, coexistence and threshold effect: The case of genetically modified herbicide tolerant oilseed rape (Brassicus napus). Ecological Modelling, 205:169180, 2007. 
[12] N. Colbach. How to model and simulate the effects of cropping systems on population dynamics and gene flow at the landscape level. Example of oilseed rape volunteers and their role for co-existence of GM and nonGM crops. Environmental Sciences \& Pollution Research, 15, 2008.

[13] Nathalie Colbach, Hervé Monod, and Claire Lavigne. A simulation study of the medium-term effects of field patterns on cross-pollination rates in oilseed rape (brassica napus 1.). Ecological Modelling, 220(5):662 $-672,2009$.

[14] C. Damgaard and G. Kjellsson. Gene flow of oilseed rape (Brassica napus) according to isolation distance and buffer zone. Agriculture, Ecosystems \&J Environment, 108(4):291-301, 2005.

[15] Michael J. de Smith, Michael F. Goodchild, and Paul A. Longley. Geospatial Analysis - A Comprehensive Guide to Principles, Techniques and Software Tools. Troubador Publishing Ltd, 2nd edition, 2007. 490 pages.

[16] R.H. Gardner and R.V. O'Neill. Pattern, Process, and Predictability: The Use of Neutral Models for Landscape Analysis. In Turner and Gardner [44], chapter 11, pages 289-307.

[17] Robert H. Gardner, Bruce T. Milne, Monica G. Turner, and Robert V. O'Neill. Neutral models for the analysis of broad-scale landscape pattern. Landscape Ecology, 1(1):19-28, 1987.

[18] C. Gaucherel. Neutral models for polygonal landscapes with linear networks. Ecological Modelling, 219:39-48, 2008.

[19] C. Gaucherel, D. Fleury, D. Auclair, and P. Dreyfus. Neutral models for patchy landscapes. Ecological Modelling, 197(1-2):159-170, 2006.

[20] C. Gaucherel, N. Giboire, V. Viaud, T. Houet, J. Baudry, and F. Burel. A domain-specific language for patchy landscape modelling: The brittany agricultural mosaic as a case study. Ecological Modelling, 194(13):233-243, 2006. Special Issue on the Fourth European Conference on Ecological Modelling.

[21] C. A. Gotway and B. M. Rutherford. The components of geostatistical simulation. In 2nd International symposium on spatial accuracy assessment in natural resources and environmental sciences, Fort Collins (CO), 1996. 
[22] W.W. Hargrove, F.M. Hoffman, and P.M. Schwartz. A fractal landscape realizer for generating synthetic maps. Conservation Ecology, 6(1):2, 2002 .

[23] D.T. Haydon and E.R. Pianka. Metapopulation theory, landscape models, and species diversity. Ecoscience, 6:316-328, 1999.

[24] E. Klein, C. Lavigne, H. Picault, R. Michel, and P.-H. Gouyon. Pollen dispersal of oilseed rape: estimation of the dispersal function and effects of field dimension. Journal of Applied Ecology, 43(1):141-151, 2006.

[25] E.K. Klein, C. Lavigne, X. Foueillassar, P.-H. Gouyon, and C. Laredo. Corn pollen dispersal: quasi-mechanistic models and field experiments. Ecological Monographs, 73:131-150, 2003.

[26] W.A. Kurz, S.J. Beukama, W. Klenner, J.A. Greenough, D.C.E Robinson, A.D. Sharpe, and T.M. Webb. Telsa: the tool for exploratory landscape scenario analyses. Computers and Electronics in Agriculture, 27:227-242, 2000 .

[27] Claire Lavigne, Etienne K. Klein, J.-F. Mari, Florence Le Ber, Katarzyna Adamczyk, Hervé Monod, and Frédérique Angevin. How do genetically modified (GM) crops contribute to background levels of GM pollen in an agricultural landscape? Journal of Applied Ecology, 45(4):1104-1113, 2008.

[28] F. Le Ber and M. Benoît. Modelling the spatial organisation of land use in a farming territory. Example of a village in the Plateau Lorrain. Agronomie: Agriculture and Environment, 18:101-113, 1998.

[29] F. Le Ber, M. Benoît, C. Schott, J.-F. Mari, and C. Mignolet. Studying crop sequences with CARROTAGE, a HMM-based data mining software. Ecological Modelling, 191(1):170-185, 2006.

[30] Florence Le Ber, Claire Lavigne, J.-F. Mari, Katarzyna Adamczyk, and Frédérique Angevin. GenExP, un logiciel pour simuler des paysages agricoles, en vue de l'étude de la diffusion de transgènes. In C. Weber and P. Gançarski, editors, Actes du Colloque International de Géomatique et d'Analyse Spatiale (SAGEO 2006), Strasbourg, pages 1-12, 2006. Actes sur CD.

[31] B. Lécroart, A. Gauffreteau, M. Le Bail, M. Leclaire, and A. Messéan. Coexistence of gm and non-gm maize: effect of regional structural variables on gm dissemination risk. In Third international conference on 
Coexistence between Genetically Modified and non GM based supply chains, pages 115-118, Sevilla, 2007. European communities.

[32] H. Li and J.F. Reynolds. A simulation experiment to quantify spatial heterogeneity in categorical maps. Ecology, 75(8):2446-2455, 1994.

[33] J. Liu and P. S. Ashton. FORMOSAIC: an individual-based spatially explicit model for simulating forest dynamics in landscape mosaics. Ecological Modelling, 106:177-200, 1998.

[34] M.S. Mackisack and R.E. Miles. Homogeneous rectangular tessellations. Advances in Applied Probability, 28:993-1013, 1996.

[35] J.-F. Mari and F. Le Ber. Temporal and Spatial Data Mining with Second-Order Hidden Markov Models. Soft Computing - A Fusion of Foundations, Methodologies and Applications, 10(5):406-414, 2006.

[36] A. Messéan, F. Angevin, M. Gómez-Barbero, K. Menrad, and E. Rodríguez-Cerezo. New case studies on the coexistence of gm and non-gm crops in european agriculture. Technical Report Series of the Joint Research Center of the European Commission, 2006. EUR 22102 En.

[37] W. Nagel and V. Weiss. The crack tessellation -characterization of the stationary random tessellations which are stable with respect to iteration. Advances in Applied Probability, 2005.

[38] Atsuyuki Okabe, Barry Boots, Kokichi Sugihara, and Sung Nok Chiu. Spatial tessellations: concepts and applications of Voronoi diagrams. John Wiley \& Sons, Inc., 2nd edition, 2000.

[39] J. O'Rourke. Computational Geometry in C. Cambridge University Press, 1998. 2nd edition.

[40] K.H. Riitters, R.V. O'Neil, C.T. Hunsaker, J.D. Wickham, D.H. Yankee, S.P. Timmins, K.B. Jones, and B.L. Jackson. A factor analysis of landscape pattern and structure metrics. Landscape ecology, 10(1):2339, 1995.

[41] O. Sanvido, F. Widmer, M. Winzeler, B. Streit, E. Szerencsits, and F. Bigler. Konzept für die Koexistenz verschiedener landwirtschaftlicher Anbausysteme mit und ohne Gentechnik in der Schweiz. Schriftenreihe der FAL Nr. 55 Agroscope FAL Reckenholz. Swiss Federal Research Station for Agroecology and Agriculture, Zurich, 2005. 
[42] S. Saura and J. Martínez-Millán. Landscape patterns simulation with a modified random clusters method. Landscape Ecology, 15:661-678, 2000 .

[43] K. Tolstrup, S. Andersen, B. Boelt, M. Buus, M. Gylling, P. Bach Hom, G. Kjellson, S. Pedersen, H. Østergård, and S. Mikkelsen. Report from the Danish working group on the coexistence of genetically modified crops with conventional and organic crops. DIAS report $n^{\circ} 94$. Ministry of Food, Agriculture and Fisheries - Danish Institute of Agricultural Sciences, 2003.

[44] M. G. Turner and R. H. Gardner, editors. Quantitative Methods in Landscape Ecology, volume 82 of Ecological Studies. Springer, 1991.

[45] M.N.M van Lieshout, editor. Markov point processes and their applications. Imperial College Press, 2000.

[46] V. Viaud, C. Lavigne, H. Monod, F. Angevin, and K. Adamczyk. Spatial sensivity of maize gene-flow from genetically modified to conventional varieties to landscape pattern: a simulation approach. Landscape Ecology, 23:1067-1079, 2008.

[47] Kimberly A. With and Anthony W. King. The use and misuse of neutral landscape models in ecology. Oikos, 79(2):219-229, 1997.

[48] J. Wu and S. A. Levin. A patch-based spatial modeling approach: conceptual framework and simulation scheme. Ecological Modelling, 101(1997):325-346, 1997. 